\title{
Estimación de la estatura a través de la tibia en población contemporánea española adulta femenina
}

\author{
Estimation of stature from tibia length in female adult \\ contemporary Spanish population
}

\begin{abstract}
Resumen
La estatura es un parámetro importante para el estudio e identificación de individuos a partir de restos óseos. Es una característica poblacional e individual, exclusiva de la especie humana, en la que intervienen factores diversos como la genética, el sexo o la alimentación. Dada la relación que existe entre la estatura de una persona y la longitud de sus huesos largos, en este estudio se propone un método de estimación de la estatura en base a la longitud de la tibia, en población española contemporánea femenina. La muestra de estudio está compuesta por las medidas de talla y tibia de 35 cadáveres de mujeres de acreditada procedencia peninsular, sin ningún factor de alteración de estos parámetros (fracturas, patologías que afecten al crecimiento, etc.).
\end{abstract}

Se ha obtenido una fórmula que mejora la estimación que proporciona el método de Telkka, sobre todo en individuos de estatura menor o igual a $150 \mathrm{~cm}$. No es necesario hacer correcciones de edad.

Palabras clave: Antropología forense. Tibia. Estatura. Osteometría. Población española femenina. Identificación.

\section{Abstract}

The stature is an important parameter for the study and identification of individuals from skeletal remains. It is a population and individual characteristic, a parameter unique to the human species in the various factors involved such as genetics, sex, or feeding. Given the relationship between a person's height and length of their long bones, this study proposes a method of estimation of stature based in the length of the tibia in female Spanish population. The sample consists of the measures of height and tibia of 35 female cadavers of Spanish origin acredited, without any alteration of these factors parameters (fractures, pathologies affecting growth, etc.).

A formula that improves the estimation methods provided by Telkka, especially in individuals of height less or equal to $150 \mathrm{~cm}$. It isn't necessary to make corrections for age.

Key words: Forensic science. Stature. Tibia. Osteometry. Female Spanish population. Forensic anthropology.

\section{Introducción}

La aportación a la identificación que realiza la antropología forense puede establecerse en múltiples circunstancias: grandes catástrofes, hallazgo de restos, accidentes aéreos, víctimas de acciones violentas, personas desaparecidas o perdidas, etc. ${ }^{1}$. En antropología identificar es comparar y, por tanto, es posible realizar una identificación positiva si tenemos elementos para la comparación: es decir, si tenemos registros, fotografías, datos clínicos de patología ósea previa, estudios radiológicos, dentales, etc., que nos permitan comparar los resultados obtenidos en laboratorio y saber si los restos que estudiamos corresponden o no a la persona que se está tratando de identificar ${ }^{2}$. Esta comparación es importante para la estatura ya que es una característica no sólo poblacional, sino también individual; puede ayudar en la identificación de restos óseos y además es un parámetro exclusivo de la especie humana (debido a la postura erguida). También es complejo y variable, en
MT. Belmonte ${ }^{1}$

JL. Sánchez Blanque ${ }^{1}$

I. Alemán ${ }^{2}$

MC. Botella ${ }^{2}$

${ }^{1}$ Instituto de Medicina Legal de Almería.

${ }^{2}$ Laboratorio de

Antropología.

Universidad de Granada.

Correspondencia:

E-mail:

antropologiaforense@

hotmail.com
Fecha de recepción: 20.NOV.2010

Fecha de aceptación: 3.JUN.2011 
el que intervienen muchos factores: genéticos (herencia multifactorial), sexo (es un parámetro biológico de dimorfismo sexual puesto que en todos los grupos humanos, la estatura de la mujer suele ser inferior a la del hombre), edad, condiciones ambientales como la alimentación, la situación socioeconómica, el medio geográfico, etc. ${ }^{1,3-8}$. Por otro lado, está influido por enfermedades óseas (patologías), e incluso es cambiante dentro de un mismo individuo en diferentes momentos del día ${ }^{4-7}$. La relación entre la estatura de un individuo y la longitud de sus huesos largos permite calcular la talla a partir de éstos. Tradicionalmente, y dada su indudable utilidad práctica, se han utilizado las tablas de Manouvrier $(1893)^{9}$, elaboradas mediante medidas tomadas de individuos franceses, y las tablas y fórmulas regresivas de Trotter y Gleser $(1952,1958,1970)^{1,9,10}$ con población americana. Diversos autores han señalado que las estimaciones de la estatura que se realizan, teniendo como base la medida de los huesos largos de las extremidades inferiores (fémur, tibia, peroné), son más precisas que las estimaciones realizadas con huesos largos de las superiores (húmero, cúbito, radio) ${ }^{8,11}$. Concretamente, Mendonça (1998)5,6 señala que en la población portuguesa, la estimación de la estatura con la longitud del fémur es más correcta que con la del húmero. Bhavna y Surinder Nath (2007) ${ }^{12}$, en su estudio con población india, señalan que la tibia es el hueso que proporciona mejores estimaciones con una menor desviación estándar y un coeficiente de correlación $r$ mayor que el resto de los huesos estudiados. Meadows y Jantz $(1999)^{8}$, con población americana, hallaron una relación más estrecha entre la estatura y longitud de tibia o peroné, que entre estatura y longitud de fémur. Agnihotri, et al. (2009) ${ }^{13}$ igualmente concluyeron en su estudio con población indomauritana, que la tibia tiene una correlación más fuerte con la estatura que el cúbito.

Diversos autores advierten sobre la precaución que hay que tener al aplicar sobre una población métodos confeccionados con otra, dadas las diferencias poblacionales existentes en relación con este parámetro ${ }^{7,13-16}$. En este sentido se evidencia la ausencia de un método específico de estimación de la estatura en población española, que tiene unas características históricas y demográficas propias que influyen en el estudio y evolución de este parámetro, puesto que, según Martínez Carrión ${ }^{17}$ el crecimiento económico moderno que tuvo lugar en España en la segunda mitad del siglo XIX no estuvo relacionado con mejoras en los niveles de vida y del bienestar, lo cual apoya la idea de que España responde a la tesis Kuznetsiana según la cual el proceso de industrialización provocó el aumento de desigualdades en la renta per cápita; y las Guerras Mundiales y Civil Española del siglo XX afectaron a los niveles de vida, acceso a alimentos, salud, etc., factores directamente relacionados, entre otros, con la estatura final que alcanza una persona.

Algunos autores han constatado un cambio general en la proporción de los segmentos corporales en las poblaciones actuales con respecto a las más antiguas $^{5,8}$, hecho que limita el uso de aquellos métodos que fueron elaborados con estas poblaciones antiguas $^{15,18}$, de ahí la importancia de este trabajo, que estudia población actual.

En la actualidad, en la antropología forense americana se utiliza el programa informático FORDISC 3.0 (Jantz y Ousley, 1993-2005), que se nutre de la Forensic Anthropology Data Bank (que contiene muestras representativas de los diferentes grupos étnicos que conviven en Estados Unidos) para proporcionar estimaciones de sexo, origen poblacional y fórmulas regresivas para estimar la estatura ${ }^{14,15,19-21}$. Aunque esta aplicación posee una indudable utilidad práctica para los grupos poblacionales representados en aquella base de datos, no es así para nuestra población de estudio, puesto que no existe en ella muestra española ${ }^{14}$. Por ello, está limitado su uso en este tipo de población y se requieren estudios poblacionales específicos ${ }^{14,19,21}$. La revisión bibliográfica nos muestra que son muchas las poblaciones en las que se ha estudiado de forma específica el comportamiento de este parámetro con respecto a la longitud de la tibia, como por ejemplo Kieffer (2010), que realizó su estudio con muestra norteamericana femenina ${ }^{22}$; Duyar y Pelin (2003) y Duyar, Pelin y Zagyapan (2006), que estudiaron población turca masculina ${ }^{23,24}$; Didia, et al. (2009), con población nigeriana de ambos sexos ${ }^{25}$; Mantilla Hernández, et al. (2009), en población colombiana masculina ${ }^{26}$; Petrovecki, et al. (2007), con población croata de ambos sexos ${ }^{11}$; Sarajlic, et al. (2006), que estudiaron población bosnia masculina ${ }^{18}$; 0 Radoinova, et al. (2002), que se basaron en población búlgara $^{27}$

Además de en el origen, estos estudios difieren en el tipo de muestra que utilizan: sujetos vivos ${ }^{12,13,22-24,26}$, cadáveres $5,6,11,18,27$ o muestra esqueletizada ${ }^{15,16}$. Algunos de ellos hacen uso de la imagen radiológica para la toma de las medidas de la tibia $^{11,18,22}$.

Esta información constata la ausencia de trabajos con las características de este proyecto, es decir, que estudie población española femenina y se base en medidas de estatura y longitud de tibia de cadáveres en fresco. 


\section{Objetivo}

El presente estudio tiene por objetivo la obtención de una fórmula regresiva para la determinación de la estatura en población española femenina actual, utilizando la longitud de la tibia como variable.

\section{Material y método}

Se han obtenido las medidas generales de la estatura y la longitud máxima de la tibia (medida 1.b de Martin, citada en Capecchi y Messeri, 197928) de 35 cadáveres femeninos de edades comprendidas entre los 27 y los 85 años, a los que se les ha practicado autopsia médico-legal en los Institutos de Medicina Legal de Almería y Granada. A través del estudio de los apellidos hasta tercera generación, se ha acreditado el origen peninsular de la muestra con objeto de homogeneizarla, por ello se han descartado a aquellas personas no originarias de la Península Ibérica. Posteriormente se tomaron las mismas medidas en otros 18 cadáveres femeninos con las mismas características a modo de controles para evaluar la efectividad de la fórmula.

En ambos casos se han desestimado también a aquellos sujetos con patología que afectara al crecimiento o patología del sistema esquelético antigua o reciente, que pudieran alterar el rigor del procedimiento y falsear la medida de la estatura o de la tibia, entre ellos cabe destacar cadáveres de personas con traumatismos craneales, vertebrales o en las extremidades, que modificaran la continuidad de los huesos, cadáveres con patologías osteo-articulares o casos de patología previa que afectara al crecimiento (hipotiroidismo, gigantismo, etc.).

\section{Técnica de medición de la estatura}

La estatura del cadáver (en centímetros) se ha obtenido midiendo con un tallímetro, siempre en decúbito supino sobre la mesa de autopsias, desde el vértex craneal al talón. Se ha procurado que el cuerpo estuviera totalmente alineado, sin ninguna diagonalidad que pudiera alterar la medida.

\section{Técnica de medición de la tibia}

Se ha tomado la medida 1.b de Martin ${ }^{28}$ de la tibia del lado izquierdo (en centímetros). Para ello se han realizado dos incisiones en el miembro inferior, una para separar el menisco de la meseta tibial, dejando liberado el cóndilo lateral, y otra dejando al descubierto y libre de tejido blando el maléolo tibial. Se han introducido dos escuadras rígidas en sendas incisiones de forma que queden totalmente paralelas entre ellas y perpendiculares con respecto al plano horizontal. Por último se ha obtenido la distancia entre ambas escuadras con una regla milimetrada. Para asegurar la ausencia total de diagonalidad en la medición, se ha utilizado un nivel básico.

\section{Resultados}

Se han analizado estadísticamente las medidas de la estatura y de la tibia (Iongitud máxima) tomadas de los 35 cadáveres, para ello se ha utilizado el programa SPSS 15.0. Los parámetros estadísticos básicos obtenidos se recogen en la Tabla 1.

Posteriormente se ha aplicado un análisis de correlación, en el que se ha elegido el modelo lineal por ser el que proporciona mejores datos de la relación entre la variable independiente (longitud de la tibia) y la dependiente (estatura).

\begin{tabular}{|c|c|c|c|c|c|}
\hline & $N$ & Minimo & Máximo & Media & Desv. tip. \\
\hline Edad & 35 & 27 & 85 & 51,46 & 14,981 \\
Estatura & 35 & 148,00 & 169,40 & 156,7714 & 6,21566 \\
Tibia & 35 & 29,60 & 37,90 & 33,2571 & 2,06259 \\
\hline
\end{tabular}

Tabla 1.

Estadísticos descriptivos.

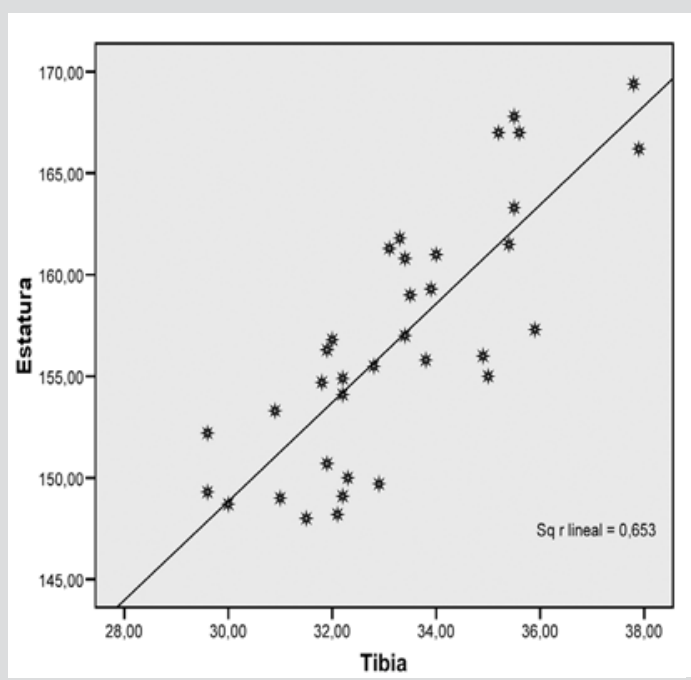

Figura 1. 
Se ha obtenido un coeficiente de correlación de Pearson $(r)$ de 0.808 entre estatura y longitud de la tibia y un $\mathrm{R}^{2}$ de 0.653 . Estos valores indican que la longitud de la tibia es una variable que puede aportar una información muy aproximada sobre la longitud total de los individuos. De igual forma, se han relacionado ambos parámetros mediante un gráfico de puntos de dispersión lineal (Figura 1).

Como se observa en la Figura 1, existe una relación lineal regresiva entre los dos parámetros. Si se aplica el modelo de fórmula regresiva, $y=a+b x$ a los datos referidos, y añadiendo el diferencial $( \pm$ k), obtenemos la siguiente ecuación para la obtención de la estatura del sujeto:

$$
E=75.798+2.435 T \pm 7.2 \mathrm{~cm}
$$

Donde $\mathrm{E}$ es la estatura (en $\mathrm{cm}$ ) y $\mathrm{T}$ es la longitud de la tibia (longitud máxima, en $\mathrm{cm}$ ).

Al aplicar la fórmula obtenida en 18 casos, obtenemos que la fórmula proporciona estimaciones correctas en el $100 \%$ de los casos, es decir, engloban la estatura real tomada del cadáver en el rango mínimo y máximo que propone.
No es necesario hacer correcciones por la edad puesto que la aplicación de la fórmula es satisfactoria dentro del amplio rango de edad de los sujetos que componen la muestra de control, cuyos parámetros estadísticos básicos obtenidos mediante el programa SPSS se recogen en la Tabla 2.

Este programa ha permitido realizar un t-test para muestras pareadas, relacionando la estatura real de los 18 casos de control y la estatura estimada por la fórmula que se propone con el fin de observar la bondad de la misma. Se ha obtenido una correlación de 0,827 entre ambas variables, con una diferencia de medias entre ellas de 0.29778 centímetros, con un intervalo de confianza al $95 \%$ de $(-1.50331$; 2.09887 ) y un nivel de significación $P=0.732$, lo cual indica que no existen diferencias significativas entre la estatura estimada por la fórmula y la estatura real de los 18 casos estudiados. Estos datos se recogen en las Tablas 3-5.

Al representar gráficamente mediante un diagrama de puntos (Figura 2) la estatura real de los casos de control y la estatura neta estimada por la fórmula (sin añadir diferencial), no se observan desviaciones claras hacia ningún extremo del intervalo.

Estadísticos descriptivos (muestra de control).

\begin{tabular}{|c|c|c|c|c|c|}
\hline & $N$ & Minimo & Máximo & Media & Desv. tip. \\
\hline Edad & 18 & 25 & 85 & 52,59 & 15,541 \\
Estatura & 18 & 141,00 & 165,00 & 155,8556 & 6,44024 \\
Tibia & 18 & 29,20 & 37,00 & 32,7556 & 2,18018 \\
\hline \multicolumn{7}{|c}{ Prueba T } \\
\hline
\end{tabular}

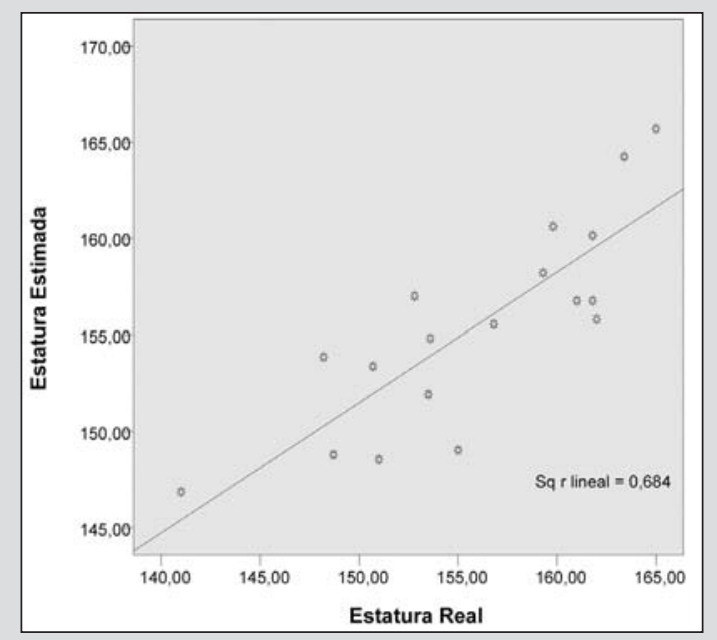

Tabla 3.

Estadísticos de muestras relacionadas.

\begin{tabular}{|cc|c|c|c|c|}
\hline & & Media & $N$ & $\begin{array}{c}\text { Desviación } \\
\text { tip. }\end{array}$ & $\begin{array}{c}\text { Error tip. de } \\
\text { la media }\end{array}$ \\
\hline \multirow{2}{*}{ Par 1 } & Estatura & 155,8556 & 18 & 6,44024 & 1,51798 \\
& Estimada & 155,5578 & 18 & 5,30875 & 1,25128 \\
\hline
\end{tabular}

Tabla 4. Correlaciones de muestras relacionadas.

\begin{tabular}{|ll|c|c|c|}
\hline & & $N$ & Correlación & Sig. \\
\hline Par 1 Estatura y Estimada & 18 &, 827 &, 000 \\
\hline
\end{tabular}

\section{Figura 2}

Tabla 5. Prueba de muestras

\begin{tabular}{|c|c|c|c|c|c|c|c|c|c|}
\hline & \multicolumn{5}{|c|}{ Diferencias relacionadas } & \multirow{3}{*}{$t$} & \multirow{3}{*}{$g l$} & \multirow{3}{*}{$\begin{array}{c}\text { Sig. } \\
\text { (bilateral) }\end{array}$} \\
\hline & & \multirow[t]{2}{*}{ Media } & \multirow{2}{*}{$\begin{array}{c}\text { Desviación } \\
\text { típ. }\end{array}$} & \multirow{2}{*}{$\begin{array}{l}\text { Error típ. de } \\
\text { la media }\end{array}$} & \multicolumn{2}{|c|}{$\begin{array}{l}\text { 95\% Intervalo de confianza } \\
\text { para la diferencia }\end{array}$} & & & \\
\hline & & & & & Superior & Inferior & & & \\
\hline Par 1 & $\begin{array}{l}\text { Estatura - } \\
\text { Estimada }\end{array}$ & 29778 & 3,62182 & ,85367 & $-1,50331$ & 2,09887 & 349 & 17 & ,732 \\
\hline
\end{tabular}


Para la aplicación de esta fórmula se debe tener en cuenta la diferencia de $2 \mathrm{~mm}$ que existe entre hueso fresco y hueso seco, en el que no existe cartílago articular, como constataron autores como Rollet (1888) y Manouvrier (1892) $)^{5,6,16}$; los mismos autores establecen una diferencia de $2 \mathrm{~cm}$ entre la talla de una persona viva y su cadáver, siendo ésta mayor por la ausencia de compresión de discos intervertebrales que tiene lugar en la posición bípeda, lo que también debe ser tenido en cuenta en la aplicación práctica de la fórmula que propone este estudio. Así pues, debe restarse 2 centímetros a la estatura máxima y mínima que proporciona la fórmula que se propone.

\section{Discusión}

La antropología forense en España no cuenta con macrobases de datos antropológicas como posee la disciplina en América. Por este motivo, y por la variación poblacional de los parámetros antropológicos, se hace necesario este tipo de estudios con poblaciones concretas y muestra actual.

Al aplicar el método de Telkka ${ }^{5,9}$ sobre la muestra de estudio ( $\mathrm{N}=35)$, se observan problemas de estimación especialmente en los casos de estatura menor o igual a $150 \mathrm{~cm}$ (que constituyen un $22,85 \%$ de la muestra total). Dicho método sólo proporciona estimaciones correctas (la estatura real del sujeto queda englobada en los rangos máximo y mínimo que dicha fórmula propone) en un $37,5 \%$ de los casos de estatura menor o igual a $150 \mathrm{~cm}$, constatándose un error de hasta $2,12 \mathrm{~cm}$. Con respecto a los casos de estatura mayor a $150 \mathrm{~cm}(77,4 \%$ de la muestra total), el método de Telkka proporciona estimaciones correctas en un $77,77 \%$ de los casos, con un error de hasta $1,9 \mathrm{~cm}$.

Lo cual evidencia las precauciones a tener en cuenta a la hora de aplicar sobre una población métodos confeccionados con otra ${ }^{7,13-16}$.

Al aplicar la fórmula propuesta en este estudio sobre 18 casos de control, se obtienen estimaciones correctas en el $100 \%$ de los mismos, es decir, la estatura real queda incluida en el rango de estatura mínima y máxima que propone. Los parámetros estadísticos que se han obtenido mediante un t-test que relaciona la estatura real de los casos control y la estatura estimada por la fórmula propuesta, indican que tiene aplicabilidad práctica y proporciona resultados fiables en la estimación de la estatura.

Los resultados de aplicar sobre la muestra la fórmula de Trotter y Gleser para la tibia no son comparables puesto que existe duda sobre si las autoras incluían o no el maleólo tibial en la medida que efectuaban de la longitud del hueso. Jantz, et al. (1994 y $1995)^{29,30}$ defienden que no lo incluyeron aunque en la definición proporcionada por las propias autoras ${ }^{1}$ se considere que sí.

También propusieron la aplicación de un factor de corrección ${ }^{1}$ para sujetos mayores de 30 años, por considerar que a partir de esa edad empieza la disminución de la estatura total del individuo por efecto de la edad a razón de $1 \mathrm{~mm}$ por año. Para Reverte $\mathrm{Coma}^{9}$, el proceso de regresión de la estatura se inicia entre los 40 y 60 años de edad y se acelera entre los 65 y los 70 años. Para este autor, la talla disminuye $0,06 \mathrm{~cm}$ por año. Otros autores como Giles (1991, citado en Krenzer, 20064) señalan que la disminución es más acusada en mayores de 70 años.

En este estudio, no se hace necesaria la introducción de un factor de corrección de la edad puesto que la fórmula proporciona estimaciones correctas en todos los casos, incluidos los casos de edad superior a 40 años ( $72 \%$ de los casos de control).

\section{Conclusiones}

La tibia es un hueso largo que proporciona resultados satisfactorios para la determinación de la estatura, tal y como se demuestra en el presente estudio, en el que se ha obtenido un coeficiente de correlación de Pearson ( $r$ ) de 0.808 entre ambas variables. La muestra objeto de estudio, al tratarse íntegramente de mujeres de origen español, ofrece una valiosa información sobre el comportamiento de este parámetro en población femenina actual, puesto que los métodos tradicionalmente utilizados están basados en población antigua, y se han constatado cambios en las proporciones corporales a lo largo del tiempo en las diferentes poblaciones, lo cual exige actualización y adecuación de los métodos a poblaciones actuales.

El método propuesto mejora la estimación de la estatura que realiza el método de Telkka en todos los rangos de este parámetro, especialmente en los casos de estatura menor o igual a $150 \mathrm{~cm}$. No es comparable con el método de Trotter y Gleser puesto que existe duda sobre si las autoras incluyeron el maléolo tibial en la medida de la longitud de la tibia en la que basaron sus estudios.

Proporciona estimaciones de la estatura fiables en todos los casos de control sobre los que ha sido aplicado, y los resultados del test de muestras pareadas realizado dan cuenta de su utilidad práctica.

No es necesario introducir un factor de corrección por la edad. 


\section{Agradecimientos}

A las doctoras Dña. Elisa Cabrerizo Medina y Dña. María Teresa Miranda por su inestimable ayuda en la elaboración de este trabajo.

\section{Bibliografía}

1. Trotter M. Estimation of stature from intact long bones. En: Stewart TD. Personal Identification in Mass Disasters. Washington: Museo Nacional de Historia Natural de Washington, 1970; p. 71- 84.

2. Ramey Burns K. Manual de Antropología Forense. Barcelona: Ed. Bellaterra, 2008; p. 249-53.

3. Bogin BY, Keep R. Eight thousand years of economic and political history in Latin America revealed by anthropometry. Annals of Human Biology 1999;26(4):333- 51.

4. Krenzer U. Compendio de Métodos Antropológicos Forenses. Tomo V: Características Individualizantes. Centro de Análisis Forense y Ciencias Aplicadas. Guatemala. 2006. 4, 5,10, 11, 26.

5. De Mendonça MC. Determinación de la talla a través de la longitud de los huesos largos. Tesis Doctoral. Universidad Complutense de Madrid. 1998. 14-34, 68.

6. De Mendonça MC. Estimation of Height from de Length of Long Bones in a Portuguese Adult Population. American Journal of Physical Anthropology 2000;112:39-48.

7. Bass W. Human Osteology. A Laboratory and Field Manual. $3^{\mathrm{a}}$ edición. Columbia: Missouri Archaelogical Society, 1987; p. 22-8.

8. Jantz LM, Jantz RL. Secular change in Long Bone Length and Proportion in the United States, 18001970. American Journal of Physical Anthropology 1999;110:57-67.

9. Reverte Coma JM. Antropología Forense. Madrid Ministerio de Justicia. Centro de Publicaciones, 1991; p. 619-64.

10. Bennett KA. A field guide for human skeletal identification. Springfield: Ed. Charles C. Thomas, 1993: p. 62-78.

11. Petrovecki V, Mayer D, et al. Prediction of Stature Based on Radiographic Measurements of cadaver long bones: a study of the Croatian Population. Journal of Forensic Sciences 2007;52(3):547-52.

12. Nath SYB. Estimation of Stature on the Basis of Measurements of the Lower Limb. Anthropologist Special. 2007;3:219-22.
13. Agnihotri AK, et al. Estimating stature from percutaneous length of tibia and ulna in Indo-Mauritian population. Forensic Science International 2009;187:109e1-109e3.

14. Spradley MK, Jantz RL, Robinson A, Peccerelli F. Demographic Change and Forensic Identification: Problems in Metric Identification of Hispanic Skeletons. Journal of Forensic Sciences 2008; 53(1):21-8.

15. Wilson RJ, Herrmann NP, Jantz LM. Evaluation of stature estimation from the Database for Forensic Anthropology. Journal of Forensic Sciences 2010;55(3):684-9.

16. Nath $S$, Badkur P. Reconstruction of stature from long bone lengths. Anthropologist Special 2002;1:109-14.

17. Martínez Carrión JM. Niveles de vida y desarrollo económico en la España Contemporánea: una visión antropométrica. Revista de Historia Económica 1994;3;685-716.

18. Sarajlic N, Cihlarz Z, Klonowski AE, Selak I. Stature Estimation for Bosnian Male Population. Bosnian Journal of Basic Medical Sciences 2006;6(1):62-7.

19. Shields KJ. The Importance of individual and population variation to human stature estimation. Tesis Doctoral. Missoula, Montana (EEUU). Universidad de Montana, 1995; p. 26-35.

20. Ubelaker DH. Fordisc 2.0: Personal computer forensic discriminant functions. International journal of Osteoarchaeology 1998;8:128-33.

21. Ubelaker DH, Ross AH, Graver SM. Application of forensic Discriminant functions to a Spanish Cranial sample. Forensic Science Communications 2002;4:3.

22. Kieffer CL. Tibia and Fibula stature formulae for modern female populations based on digital radiographic measurements. Journal of Forensic Sciences 2010;55(3):695-700.

23. Duyar I, Pelin C. Body height estimation based on tibia length in different stature groups. American Journal of Physical Anthropology 2003;122:23-7.

24. Duyar I, Pelin C, Zagyapan R. A new method of stature estimation for forensic anthropological application. Anthropological Science 2006;114:23-7. 
25. Didia BC, et al. Stature Estimation Formulae for Nigerians. Journal of Forensic Sciences 2009;54(1):20-1.

26. Mantilla Hernández JC, et al. Estimación de la talla a partir de la medida de la tibia en población colombiana. International Journal Morphology 2009;27(2):305-9.

27. Radoinova $D$, et al. Stature Estimation from long bones lengths in Bulgarian. Homo 2002;52(3):221-32.
28. Capecchi V, Messeri P. Antropologia. Roma: Ed. Universo, 1979; p. 395-7.

29. Jantz RL, Hunt DR, Meadows L. Maximun Length of the Tibia: How did Trotter measure it? American Journal of Physical Anthropology 1994:93:525-8.

30. Jantz RL, Hunt DR, Meadows L. The measure and mismeasure of the tibia: implications for stature estimation. Journal of Forensic Sciences 1995:40(5):758-61. 